\begin{tabular}{|c|c|}
\hline Title & Ground State of the Massless Nel son Model Without Infrared Cutoff in a Non-Fock Representation \\
\hline Author(s) & A rai, A sao \\
\hline Citation & $\begin{array}{l}\text { Reviews in Mathematical Phy sics, 13(9), 1075-1094 } \\
\text { https://doi.org/10.1142/S0129055X 01000934 }\end{array}$ \\
\hline Issue Date & 2001-09 \\
\hline Doc URL & http:/hdl.handle.net/2115/38265 \\
\hline Rights & $\begin{array}{l}\text { Electronic version of an article published as Reviews in Mathematical Phy sics, V ol. 13, Issue 9, 2001, pp. 1075-1094, } \\
\text { DOI: } 10.1142 / 50129055 X 01000934 \text { @ W orld Scientific Publishing Company, } \\
\text { http:/www.worldscinet.com/rmp/mp.shtml }\end{array}$ \\
\hline Type & article (author version) \\
\hline File Information & Nelson.pdf \\
\hline
\end{tabular}

Instructions for use 


\title{
Ground State of the Massless Nelson Model Without Infrared Cutoff in a Non-Fock Representation
}

\author{
Asao Arai * \\ Department of Mathematics \\ Hokkaido University \\ Sapporo 060-0810 \\ Japan
}

\begin{abstract}
We consider a model of quantum particles coupled to a massless quantum scalar field, called the massless Nelson model, in a non-Fock representation of the timezero fields which satisfy the canonical commutation relations. We show that the model has a ground state for all values of the coupling constant even in the case where no infrared cutoff is made. The non-Fock representation used is inequivalent to the Fock one if no infrared cutoff is made.
\end{abstract}

Key words: Nelson's model, massless quantum field, infrared divergence, ground state, canonical commutation relations, non-Fock representation

\section{Introduction}

We consider a system of $N$ quantum particles $(N \in \mathbb{N})$ moving on the $d$-dimensional Euclidean space $\mathbb{R}^{d}(d \in \mathbb{N})$ under the influence of an external potential $V: \mathbb{R}^{d N} \rightarrow$ $\mathbb{R}$ (Borel measurable) and coupled to a massless quantum scalar field. The model we discuss here is the so-called massless Nelson model [10]. The problem to which we address ourselves in this paper is that of existence of a ground state of the model without infrared cutoff. It has been shown that the massless Nelson model with infrared cutoff has a ground state $[14,7]$. A natural question to be asked next is if the model without infrared cutoff has a ground state or not.

We remark that, generally speaking, there is some subtlety on the existence of ground states of massless quantum field models, which is due to possible infrared divergences.

*Supported by the Grant-In-Aid No. 11440036 for Scientific Research from the Ministry of Education, Science, Sports and Culture, Japan. 
Indeed, in a class of models which describe interactions of particles and massless quantum fields, it is proved or suggested that, if the time-zero fields are given by the Fock representation and no infrared cutoff is made in the interactions, then the models have no ground states, althogh it may depend on the strength of the parameters contained in the Hamiltonians [6, 13, 2, 3]. On the other hand, the Pauli-Fierz model in non-relativistic quantum electrodynamics has a ground state in the Fock representation of time-zero fields even if no infrared cutoff is made. [4, 8].

As for the massless Nelson model without infrared cutoff, Lörinczi, Minlos and Spohn [9] recently proved that, in the case $d=3$ and $N=1$, it has no ground state within the Fock space where the time-zero fields are given by the usual Fock representation of the canonical commutation relations $(\mathrm{CCR})$ indexed by $\mathcal{S}_{\text {real }}\left(\mathbb{R}^{d}\right)$, the space of real-valued, rapidly decreasing $C^{\infty}$-functions on $\mathbb{R}^{d}$.

The purpose of this paper is to point out that, if we consider the massless Nelson model in a non-Fock representation of the CCR for time-zero fields, then it has a ground state even in the case where no infrared cutoff is made. This new representation of the massless Nelson model is inequivalent to the Fock one if no infrared cutoff is made.

This paper is organized as follows. In Section 2 we first briefly review the Nelson model in the standard form in which the time-zero fields are given by the Fock representation of the CCR indexed by $\mathcal{S}_{\text {real }}\left(\mathbb{R}^{d}\right)$. We call it the "standard Nelson model" (SNM). Then we give an algebraic characterization of the Nelson model in a way independent of the choice of representations of the time-zero fields and derive rigorously field equations in a weak sense. To the author's best knowledge, field equations of the Nelson model has not been rigorously established so far (only formal or classical ones are available). In Section 3 we define the Nelson model in a non-Fock representation of the time-zero fields. In the last section, we prove that, under suitable hypotheses, the massless Nelson model introduced in Section 3 has a ground state even in the case where no infrared cutoff is made.

\section{Algebraic characterization and field equations of the Nelson model}

In this section we first recall the SNM. Then we present an algebraic characterization of the Nelson model in an abstract manner. Finally we derive rigorously field equations of the abstract Nelson model.

\section{$2.1 \quad$ The SNM}

The coordinate of the configuration space $\mathbb{R}^{d N}$ of the $N$ particles is denoted $q=\left(q_{1}, \cdots\right.$, $\left.q_{N}\right) \in \mathbb{R}^{d N}$ with $q_{j}:=\left(q_{j 1}, \cdots, q_{j d}\right) \in \mathbb{R}^{d}(j=1, \cdots, N)$. Let $D_{j \mu}(j=1, \cdots, N, \mu=$ $1, \cdots, d)$ be the generalized partial differential operator in the variable $q_{j \mu}$, so that the momentum operator of the $j$-th particle is given by $p_{j}:=\left(p_{j 1}, \cdots, p_{j d}\right)$ with $p_{j \mu}:=-i D_{j \mu}$. The Hamiltonian of the particle system is then given by the Schrödinger operator

$$
H_{\mathrm{p}}:=\sum_{j=1}^{N} \frac{p_{j}^{2}}{2 m_{j}}+V,
$$


acting on $L^{2}\left(\mathbb{R}^{d N}\right)$, where $m_{j}>0$ is the mass of the $j$-th particle and $p_{j}^{2}=\sum_{\mu=1}^{d} p_{j \mu}^{2}=$ $-\Delta_{q_{j}}$ is the $d$-dimensional generalized Laplacian in the variable $q_{j}$. For a linear operator $T$, we denote its domain by $D(T)$. We assume the following:

(H.1) The operator $H_{\mathrm{p}}$ is self-adjoint on its natural domain $D\left(H_{\mathrm{p}}\right)=\cap_{j=1}^{N} D\left(\Delta_{q_{j}}\right) \cap D(V)$ and bounded from below.

The Hilbert space for state vectors of the quantum scalar field of the SNM is given by

$$
\mathcal{F}_{\mathrm{b}}:=\oplus_{n=0}^{\infty} \otimes_{\mathrm{s}}^{n} L^{2}\left(\mathbb{R}^{d}\right),
$$

the Boson Fock space over $L^{2}\left(\mathbb{R}^{d}\right)$, where $\otimes_{\mathrm{s}}^{n} L^{2}\left(\mathbb{R}^{d}\right)$ is the symmetric tensor product of $L^{2}\left(\mathbb{R}^{d}\right)\left(\otimes_{\mathrm{s}}^{0} L^{2}\left(\mathbb{R}^{d}\right):=\mathbb{C}\right)$. Let $\omega$ be a nonnegative Borel measurable function on $\mathbb{R}^{d}$ such that $0<\omega(k)<\infty$ for almost everywehre (a.e.) $k \in \mathbb{R}^{d}$ with respect to the $d$-dimensional Lebesgue measure and

$$
\omega(k)=\omega(-k) \quad \text { a.e. } k \text {. }
$$

For a.e. $k \in \mathbb{R}^{d}, \omega(k)$ physically means the energy of one free boson with momentum $k$. The function $\omega$ defines a nonnegative self-adjoint multiplication operator on $L^{2}\left(\mathbb{R}^{d}\right)$ which is injective.

Remark 2.1 A physical example for $\omega$ is given by

$$
\omega_{m}(k):=\sqrt{k^{2}+m^{2}}, \quad k \in \mathbb{R}^{d}
$$

with $m \geq 0$ a constant denoting the mass of one boson. We are interested in the massless case $m=0: \omega_{0}(k)=|k|$

The free Hamiltonian of the quantum scalar field is defined by

$$
H_{\mathrm{b}}:=d \Gamma(\omega)
$$

the second quantization of $\omega[11, \S \mathrm{X} .7]$.

We denote the annihilation operators on $\mathcal{F}_{\mathrm{b}}$ by $a(f)=\int_{\mathbb{R}^{d}} a(k) f(k)^{*} d k\left(f \in L^{2}\left(\mathbb{R}^{d}\right)\right)$ $[11, \S \mathrm{X} .7]$. The symmetric operator

$$
\Phi_{\mathrm{S}}(f):=\frac{1}{\sqrt{2}}\left(a(f)^{*}+a(f)\right),
$$

called the Segal field operator, is essentially self-adjoint [11, §X.7]. We denote its closure by the same symbol.

Let $\mathcal{S}_{\text {real }}^{\prime}\left(\mathbb{R}^{d}\right)$ be the set of real tempered distributions on $\mathbb{R}^{d}$ and, for each $s \in \mathbb{R}$,

$$
H_{\omega}^{s}:=\left\{f \in \mathcal{S}_{\text {real }}^{\prime}\left(\mathbb{R}^{d}\right) \mid \omega^{s} \hat{f} \in L^{2}\left(\mathbb{R}^{d}\right)\right\},
$$

where

$$
\hat{f}(k):=\frac{1}{(2 \pi)^{d / 2}} \int_{\mathbb{R}^{d}} f(x) e^{-i k x} d x
$$


is the Fourier transform of $f$. For $f \in H_{\omega}^{-1 / 2}$ and $g \in H_{\omega}^{1 / 2}$, we can define

$$
\phi_{\mathrm{F}}(f):=\Phi_{\mathrm{S}}\left(\frac{\hat{f}}{\sqrt{\omega}}\right), \quad \pi_{\mathrm{F}}(g):=\Phi_{\mathrm{S}}(i \sqrt{\omega} \hat{g}) .
$$

Let

$$
\mathcal{F}_{0}:=\left\{\psi=\left\{\psi^{(n)}\right\}_{n=0}^{\infty} \in \mathcal{F}_{\mathrm{b}} \mid \psi^{(n)}=0 \text { for all but finitely many } n \text { 's }\right\},
$$

called the subspace of finite-particle vectors. Then, for all $f \in H_{\omega}^{-1 / 2}$ and $g \in H_{\omega}^{1 / 2}, \phi_{\mathrm{F}}(f)$ and $\pi_{\mathrm{F}}(g)$ leave $\mathcal{F}_{0}$ invariant satisfying the CCR

$$
\begin{aligned}
& {\left[\phi_{\mathrm{F}}(f), \pi_{\mathrm{F}}(g)\right]=i \int_{\mathbb{R}^{d}} \hat{f}(k)^{*} \hat{g}(k) d k,} \\
& {\left[\phi_{\mathrm{F}}(f), \phi_{\mathrm{F}}\left(f^{\prime}\right)\right]=0, \quad\left[\pi_{\mathrm{F}}(g), \pi_{\mathrm{F}}\left(g^{\prime}\right)\right]=0, f, f^{\prime} \in H_{\omega}^{-1 / 2}, g, g^{\prime} \in H_{\omega}^{1 / 2},}
\end{aligned}
$$

on $\mathcal{F}_{0}$. Namely, $\left\{\phi_{\mathrm{F}}(f), \pi_{\mathrm{F}}(g) \mid f \in H_{\omega}^{-1 / 2}, g \in H_{\omega}^{1 / 2}\right\}$ gives a representation of the CCR. This representation is called the Fock representation of the CCR. In the SNM, $\phi_{\mathrm{F}}(f)$ and $\pi_{\mathrm{F}}(g)$ are taken to be the time-zero fields.

The Hilbert space for the SNM is

$$
\mathcal{H}:=L^{2}\left(\mathbb{R}^{d N}\right) \otimes \mathcal{F}_{\mathrm{b}}
$$

As usual, we freely use the natural identification of $\mathcal{H}$ with $\int_{\mathbb{R}^{d N}}^{\oplus} \mathcal{F}_{\mathrm{b}} d q$, the constant fibre direct integral with base space $\left(\mathbb{R}^{d N}, d q\right)$ and fibre $\mathcal{F}_{\mathrm{b}}[12, \S$ XIII.16]. In what follows, for notational simplicity, a decomposable operator $A=\int_{\mathbb{R}^{d N}}^{\oplus} A(q) d q$ on $\mathcal{H}$ with fibre $A(q)$ (which is an operator on $\mathcal{F}_{\mathrm{b}}$ for each $q \in \mathbb{R}^{d N}$ ) is denoted $A(q)$ also.

To describe an interaction between the particles and the quantum scalar field, we fix distributions $\rho_{j} \in \mathcal{S}_{\text {real }}^{\prime}\left(\mathbb{R}^{d}\right), j=1, \cdots, N$, which satisfy the following:

(H.2) For $j=1, \cdots, N$,

$$
\rho_{j} \in H_{\omega}^{s}, \quad s=-1 / 2,-1 .
$$

The interaction of the particles and the quantum field in the SNM is given by the operator

$$
H_{I}^{\mathrm{F}}:=\sum_{j=1}^{N} \Phi_{\mathrm{S}}\left(e^{-i k q_{j}} \frac{\hat{\rho}_{j}^{*}}{\sqrt{\omega}}\right)
$$

acting in $\mathcal{H}$. Formally we have $H_{I}^{\mathrm{F}}=\sum_{j=1}^{N} \int_{\mathbb{R}^{d}} \phi_{\mathrm{F}}\left(q_{j}-x\right) \rho_{j}(x) d x$, where

$$
\phi_{\mathrm{F}}(x):=\int_{\mathbb{R}^{d}} \frac{1}{\sqrt{2(2 \pi)^{d} \omega(k)}}\left\{a(k)^{*} e^{-i k x}+a(k) e^{i k x}\right\} d k .
$$

The total Hamiltonian of the SNM is defined by

$$
H_{\mathrm{SNM}}:=H_{0}+\lambda H_{I}^{\mathrm{F}}
$$

where

$$
H_{0}:=H_{\mathrm{p}}+H_{\mathrm{b}}
$$

and $\lambda \in \mathbb{R} \backslash\{0\}$ denotes the coupling constant of the model. 
Proposition 2.1 Assume (H.1) and (H.2). Then $H_{\mathrm{SNM}}$ is self-adjoint with $D\left(H_{\mathrm{SNM}}\right)=$ $D\left(H_{0}\right)$ and bounded from below. Moreover, $H_{\mathrm{SNM}}$ is essentially self-adjoint on each core of $H_{0}$

Proof. A simple application of [1, Proposition B.2] which employs the Kato-Rellich theorem (cf. [6]).

In summary, the SNM is characterized in terms of the Hamiltonian $H_{\mathrm{SNM}}$ and the time-zero-fields $\left\{\phi_{\mathrm{F}}(f), \pi_{\mathrm{F}}(g) \mid f \in H_{\omega}^{-1 / 2}, g \in H_{\omega}^{1 / 2}\right\}$.

\subsection{An abstract definition of the Nelson model}

As is seen above, the SNM uses the Fock representation of the CCR to give its time-zero fields and its Hamiltonain. We want to define the Nelson model in a way independent of the choice of representations of the CCR for time-zero fields. A natural manner for this is to use commutation relations fulfilled by observables, i.e., to find a possible Lie algebraic structure. We shall take commutation relations in a weak sense.

We denote the inner product and the norm of a Hilbert space $\mathcal{X}$ by $\langle\cdot, \cdot\rangle_{\mathcal{X}}$ and $\|\cdot\|_{\mathcal{X}}$ respectively. But, if there is no danger of confusion, then we simply write them as $\langle\cdot, \cdot\rangle$ and $\|\cdot\|$.

Definition 2.2 Let $A$ and $B$ be densely defined linear operators on a Hilbert space $\mathcal{X}$ and $\mathcal{D}$ be a subspace of $\mathcal{X}$ such that $\mathcal{D} \subset D(A) \cap D(B) \cap D\left(A^{*}\right) \cap D\left(B^{*}\right)$. Then we define a quadratic form $[A, B]_{\mathrm{w}}^{\mathcal{D}}$ by

$$
[A, B]_{\mathrm{w}}^{\mathcal{D}}(\psi, \phi):=\left\langle A^{*} \psi, B \phi\right\rangle-\left\langle B^{*} \psi, A \phi\right\rangle, \quad \psi, \phi \in \mathcal{D} .
$$

("w" means "weak".)

Remark 2.2 If $A$ and $B$ are bounded on $\mathcal{X}$ with $D(A)=D(B)=\mathcal{X}$, then, for all dense subspaces $\mathcal{D}$ of $\mathcal{X},[A, B]_{\mathrm{w}}^{\mathcal{D}}(\psi, \phi)=\langle\psi,[A, B] \phi\rangle$ for all $\psi, \phi \in \mathcal{D}$, where $[A, B]:=A B-B A$ (the usual commutator).

Let $\mathcal{F}$ be a Hilbert space and

$$
\mathcal{K}:=L^{2}\left(\mathbb{R}^{d N}\right) \otimes \mathcal{F}=\int_{\mathbb{R}^{d N}}^{\oplus} \mathcal{F} d q .
$$

Definition 2.3 Assume (H.1) and (H.2). Let $s_{0}$ and $s_{1}$ be real constants. A Nelson model is a set $\mathbb{M}_{\text {Nelson }}:=\left\{\mathcal{K}, \mathcal{D}, L_{\lambda},\left\{\phi(f), \pi(g) \mid f \in H_{\omega}^{s_{0}}, g \in H_{\omega}^{s_{1}}\right\}\right\}$ having the following properties:

(N.1) $\mathcal{D}$ is a dense subspace of $\mathcal{K}$.

(N.2) $L_{\lambda}$ is a symmetric operator on $\mathcal{K}$ and the operator

$$
H_{\mathrm{NM}}:=H_{\mathrm{p}}+L_{\lambda}
$$

is self-adjoint $\left(D\left(H_{\mathrm{NM}}\right):=D\left(H_{\mathrm{p}}\right) \cap D\left(L_{\lambda}\right)\right)$. We call $H_{\mathrm{NM}}$ the Hamiltonian of the Nelson model $\mathbb{M}_{\text {Nelson }}$. 
(N.3) The function $\omega$ is such that

$$
\mathcal{S}_{\text {real }}\left(\mathbb{R}^{d}\right) \subset H_{\omega}^{s_{0}} \cap H_{\omega}^{s_{0}+2} \cap H_{\omega}^{s_{1}} \cap H_{\omega}^{1}
$$

and, for all $j=1, \cdots, N, \mu=1, \cdots, d$,

$$
D_{\mu} \rho_{j} \in H_{\omega}^{s_{0}}
$$

where $D_{\mu}(\mu=1, \cdots, d)$ is the generalized partial differential operator in the $\mu$-th variable $x_{\mu}$ in $x=\left(x_{1}, \cdots, x_{d}\right) \in \mathbb{R}^{d}$.

$(\mathrm{N} .4)\left\{\mathcal{K}, \mathcal{D},\left\{\phi(f), \pi(f) \mid f \in \mathcal{S}_{\text {real }}\left(\mathbb{R}^{d}\right)\right\}\right\}$ is a representation of the CCR indexed by $\mathcal{S}_{\text {real }}\left(\mathbb{R}^{d}\right)$. Namely, for all $f, g \in \mathcal{S}_{\text {real }}\left(\mathbb{R}^{d}\right), \phi(f)$ and $\pi(f)$ are self-adjoint operators on $\mathcal{K}$ with $\mathcal{D} \subset D(\phi(f) \pi(g)) \cap D(\pi(f) \phi(g)) \cap D(\phi(f) \phi(g)) \cap D(\pi(f) \pi(g))$ satisfying the CCR

$$
\begin{aligned}
& {[\phi(f), \pi(g)]=i \int_{\mathbb{R}^{d}} f(x) g(x) d x,} \\
& {[\phi(f), \phi(g)]=0, \quad[\pi(f), \pi(g)]=0}
\end{aligned}
$$

on $\mathcal{D}$ and the linearity:

$$
\phi(a f+b g)=a \phi(f)+b \phi(g), \quad \pi(a f+b g)=a \pi(f)+b \pi(g), \quad a, b \in \mathbb{R},
$$

on $\mathcal{D}$.

(N.5) Let $X=q_{j \mu}, p_{j \mu}, Y=\phi(f), \pi(f), j=1, \cdots, N, \mu=1, \cdots, d, f \in \mathcal{S}_{\text {real }}\left(\mathbb{R}^{d}\right)$. There exists a dense subspace $\mathcal{E}$ of $\mathcal{K}$ such that

$$
\mathcal{E} \subset D(X) \cap D(Y) \cap D\left(L_{\lambda}\right) \cap D\left(H_{\mathrm{p}}\right)
$$

and the following relations hold in the sense of quadratic form on $\mathcal{E}$ :

$$
\begin{aligned}
& {\left[L_{\lambda}, q_{j \mu}\right]_{\mathrm{w}}^{\mathcal{E}}=0, \quad\left[L_{\lambda}, p_{j \mu}\right]_{\mathrm{w}}^{\mathcal{E}}=i \lambda \phi\left(D_{\mu} \rho_{j}\left(q_{j}-\cdot\right)\right),} \\
& {\left[L_{\lambda}, \phi(f)\right]_{\mathrm{w}}^{\mathcal{E}}=-i \pi(f),} \\
& {\left[L_{\lambda}, \pi(f)\right]_{\mathrm{w}}^{\mathcal{E}}=i \phi\left(\omega(-i \nabla)^{2} f\right)+i \lambda \sum_{j=1}^{N}\left(\rho_{j} * f\right)\left(q_{j}\right),} \\
& {[X, Y]_{\mathrm{w}}^{\mathcal{E}}=0, \quad\left[H_{\mathrm{p}}, Y\right]_{\mathrm{w}}^{\mathcal{E}}=0,}
\end{aligned}
$$

where $\nabla=\left(D_{1}, \cdots, D_{d}\right)$ and $\rho_{j} * f$ is the convolution of $\rho_{j}$ and $f:\left(\rho_{j} * f\right)(x):=$ $\int_{\mathbb{R}^{d}} \rho_{j}(x-y) f(y) d y$.

Remark 2.3 (i) We have

$$
\left(\omega(-i \nabla)^{2} f\right)(x)=\frac{1}{(2 \pi)^{d / 2}} \int_{\mathbb{R}^{d}} \omega(k)^{2} \hat{f}(k) e^{i k x} d k, \quad f \in \mathcal{S}\left(\mathbb{R}^{d}\right) .
$$

Hence (N.3) implies that

$$
\omega(-i \nabla)^{2} f \in H_{\omega}^{s_{0}},
$$

so that $\phi\left(\omega(-i \nabla)^{2} f\right)$ is defined. (ii) It follows from (H.2) and (N.3) that, for all $f \in$ $\mathcal{S}_{\text {real }}\left(\mathbb{R}^{d}\right), \rho_{j} * f$ is a bounded continuous function on $\mathbb{R}^{d}$, so that $\rho_{j} * f\left(q_{j}\right)$ is a bounded self-adjoint multiplication operator with $D\left(\rho_{j} * f\left(q_{j}\right)\right)=\mathcal{K}$. 
Let us show that the SNM is indeed a Nelson model in the above sense. We introduce

$$
\mathcal{F}_{\omega, \text { fin }}:=\mathcal{L}\left\{\Omega_{0}, a\left(f_{1}\right)^{*} \cdots a\left(f_{n}\right)^{*} \Omega_{0} \mid n \in \mathbb{N}, f_{j} \in D(\omega), j=1, \cdots, n\right\},
$$

where $\Omega_{0}:=\{1,0,0, \cdots\} \in \mathcal{F}_{\mathrm{b}}$ is the Fock vacuum and $\mathcal{L}\{\cdots\}$ means the subspace algebraically spanned by all the vectors in the set $\{\cdots\}$, and

$$
\mathcal{D}_{0}:=C_{0}^{\infty}\left(\mathbb{R}^{d N}\right) \otimes_{\text {alg }} \mathcal{F}_{\omega, \text { fin }},
$$

where $\otimes_{\text {alg }}$ denotes algebraic tensor product.

Proposition 2.4 Assume (H.1) and (H.2). Suppose that

$$
\begin{aligned}
& \int_{|q| \leq R}|V(q)|^{2} d q<\infty, \forall R>0, \\
& \mathcal{S}_{\text {real }}\left(\mathbb{R}^{d}\right) \subset H_{\omega}^{3 / 2} \cap H_{\omega}^{-1 / 2},
\end{aligned}
$$

and, for $j=1, \cdots, N, \mu=1, \cdots, d$,

$$
D_{\mu} \rho_{j} \in H_{\omega}^{-1 / 2}
$$

Let

$$
L_{\lambda}^{\mathrm{SNM}}:=H_{\mathrm{b}}+\lambda H_{I}^{\mathrm{F}}
$$

Then

$$
\mathbb{M}_{\mathrm{SNM}}:=\left\{\mathcal{H}, \mathcal{D}_{0}, L_{\lambda}^{\mathrm{SNM}},\left\{\phi_{\mathrm{F}}(f), \pi_{\mathrm{F}}(g) \mid f \in H_{\omega}^{-1 / 2}, g \in H_{\omega}^{1 / 2}\right\}\right\}
$$

is a Nelson model.

Proof. (N.1) is obvious. (N.2) follows from Proposition 2.1. (N.3) with $s_{0}=-1 / 2, s_{1}=$ $1 / 2$ follows from the present assumption. (N.4) follows from (2.11) and (2.12). For (N.5), we take $\mathcal{E}=\mathcal{D}_{0}$. By condition $(2.27), C_{0}^{\infty}\left(\mathbb{R}^{d N}\right) \subset D(V)$ so that $C_{0}^{\infty}\left(\mathbb{R}^{d N}\right) \subset D\left(H_{\mathrm{p}}\right)$. Hence (2.24) follows. The commutation relations in (N.5) follow from direct computations using (2.10), (2.11) and

$$
\left[H_{\mathrm{b}}, a(f)\right]=-a(\omega f), \quad\left[H_{\mathrm{b}}, a(f)^{*}\right]=a(\omega f),
$$

on $\mathcal{F}_{\omega, \text { fin }}\left(f, \omega f \in L^{2}\left(\mathbb{R}^{d}\right)\right)$.

\subsection{Field equations}

We derive field equations for the Nelson model $\mathbb{M}_{\text {Nelson }}$ in a weak sense.

Definition 2.5 Let $\mathcal{X}$ be a Hilbert space and $A(t)(t \in \mathbb{R})$ be a linear operator on $\mathcal{X}$. Suppose that there exists a dense subspace $\mathcal{D}$ of $\mathcal{X}$ such that $\mathcal{D} \subset D(A(t))$ for all $t \in \mathbb{R}$. We say that $A(t)$ is weakly differentiable on $\mathcal{D}$ if, for all $\psi, \phi \in \mathcal{D}$, the function $\langle\psi, A(t) \phi\rangle$ is differentiable in $t \in \mathbb{R}$. In that case we define a quadratic form $\mathrm{w}-d A(t) /\left.d t\right|_{\mathcal{D}}$ on $\mathcal{D}$ by

$$
\mathrm{w}-\left.\frac{d A(t)}{d t}\right|_{\mathcal{D}}(\psi, \phi):=\frac{d}{d t}\langle\psi, A(t) \phi\rangle, \quad \psi, \phi \in \mathcal{D} .
$$


Definition 2.6 Let $\mathcal{X}$ be a Hilbert space and $H$ be a self-adjoint operator on $\mathcal{H}$. Let $A$ be a delnsely defined linear operator on $\mathcal{X}$. We say that $A$ is in the set $\mathrm{A}_{H}$ if it satisfies the following (i) and (ii):

(i) There exists a dense subspace $D_{A} \subset D(H)$ such that, for all $s \in \mathbb{R}, e^{i s H} D_{A} \subset$ $D(A) \cap D\left(A^{*}\right)$.

(ii) For all $\psi \in D_{A}$, the $\mathcal{X}$-valued functions: $s \rightarrow A e^{i s H} \psi$ and $s \rightarrow A^{*} e^{i s H} \psi$ are strongly continuous on $\mathbb{R}$.

For $A \in \mathrm{A}_{H}$, we set

$$
D_{A, H}:=\mathcal{L}\left\{e^{i s H} \psi \mid \psi \in D_{A}, s \in \mathbb{R}\right\} .
$$

Lemma 2.7 Let $\mathcal{X}$ be a Hilbert space and $H$ be a self-adjoint operator on $\mathcal{X}$. Let $A$ be a densely defined linear operator on $\mathcal{X}$ and set

$$
A(t):=e^{i t H} A e^{-i t H}, \quad t \in \mathbb{R},
$$

Assume that $A \in \mathrm{A}_{H}$. Then $A(t)$ is weakly differentiable on $D_{A, H}$ and

$$
\mathrm{w}-\left.\frac{d A(t)}{d t}\right|_{D_{A, H}}=i[H, A(t)]_{\mathrm{w}}^{D_{A, H}} .
$$

Proof. Let $\psi, \phi \in D_{A, H}$. Then $\psi, \phi \in D(A(t)) \cap D\left(A(t)^{*}\right)$ for all $t \in \mathbb{R}$. For $h \in \mathbb{R} \backslash\{0\}$,

$$
\begin{aligned}
\left\langle\psi, \frac{A(t+h)-A(t)}{h} \phi\right\rangle=\langle & \left.e^{-i t H} \frac{e^{-i h H}-1}{h} \psi, A e^{-i(t+h) H} \phi\right\rangle \\
& +\left\langle A(t)^{*} \psi, \frac{e^{-i h H}-1}{h} \phi\right\rangle .
\end{aligned}
$$

Since $\psi \in D(H)$, it follows that

$$
\lim _{h \rightarrow 0} \frac{e^{-i h H}-1}{h} \psi=-i H \psi .
$$

It follows from Definition 2.6(ii) that $A e^{i s H} \phi$ is strongly continuous in $s$. Hence

$$
\lim _{h \rightarrow 0} A e^{-i(t+h) H} \phi=A e^{-i t H} \phi .
$$

Hence

$$
\lim _{h \rightarrow 0}\left\langle\psi, \frac{A(t+h)-A(t)}{h} \phi\right\rangle=i\left\langle e^{-i t H} H \psi, A e^{-i t H} \phi\right\rangle-i\left\langle A(t)^{*} \psi, H \phi\right\rangle .
$$

Thus the desired result follows.

The canonical Heisenberg operators of the Neson model $\mathbb{M}_{\text {Nelson }}$ (Definition 2.3) are defined as follows:

$$
\begin{aligned}
& q_{j \mu}(t):=e^{i t H_{\mathrm{NM}}} q_{j \mu} e^{-i t H_{\mathrm{NM}}}, \quad p_{j \mu}(t):=e^{i t H_{\mathrm{NM}}} p_{j \mu} e^{-i t H_{\mathrm{NM}}}, \\
& \phi(t, f):=e^{i t H_{\mathrm{NM}}} \phi(f) e^{-i t H_{\mathrm{NM}}}, \quad \pi(t, f):=e^{i t H_{\mathrm{NM}}} \pi(t, f) e^{-i t H_{\mathrm{NM}}}, \\
& j=1, \cdots, N, \mu=1, \cdots, d, \quad f \in \mathcal{S}_{\text {real }}\left(\mathbb{R}^{d}\right), t \in \mathbb{R} .
\end{aligned}
$$

For a self-adjoint operator $T, Q(T)$ denotes the form domain of the quadratic form associated with $T$. 
Theorem 2.8 Consider the Nelson model $\mathbb{M}_{\text {Nelson }}$ (Definition 2.3). Assume (H.1), (H.2) and the following (A.1)-(A.5):

(A.1) (2.27) holds and $H_{\mathrm{p}}$ is essentially self-adjoint on $C_{0}^{\infty}\left(\mathbb{R}^{d N}\right)$.

(A.2) For all $j=1, \cdots, N, \mu=1, \cdots, d$, the distributional partial derivative $D_{j \mu} V$ in the variable $q_{j \mu}$ is a Borel measurable function on $\mathbb{R}^{d N}$ which is a.e. finite with respect to the Lebesgue measure. Moreover, $C_{0}^{\infty}\left(\mathbb{R}^{d N}\right)$ is a form core of the self-adjoint multiplication operator $D_{j \mu} V$ and $D\left(H_{\mathrm{p}}\right) \subset \cap_{j=1}^{N} \cap_{\mu=1}^{d} Q\left(D_{j \mu} V\right)$.

(A.3) In addition to (2.24),

$$
\mathcal{E} \subset D\left(H_{\mathrm{p}}\right) \cap\left\{\cap_{j, l=1}^{N} \cap_{\mu, \nu=1}^{d}\left[D\left(p_{j \mu} q_{l \nu}\right) \cap D\left(q_{l \nu} p_{j \mu}\right)\right]\right\} \cap\left[\cap_{j=1}^{N} \cap_{\mu=1}^{d} Q\left(D_{j \mu} V\right)\right] .
$$

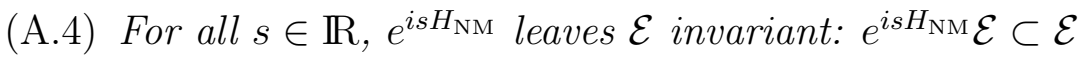

(A.5) For all $j=1, \cdots, N, \mu=1, \cdots, d$ and $\psi \in \mathcal{E}$, the $\mathcal{K}$-valued function $q_{j \mu} e^{i s H_{\mathrm{NM}}} \psi$ is strongly continuous in $s \in \mathbb{R}$.

(A.6) For all $f \in \mathcal{S}_{\text {real }}\left(\mathbb{R}^{d}\right)$, the $\mathcal{K}$-valued functions $\phi(f) e^{i s H_{\mathrm{NM}}} \psi$ and $\pi(f) e^{i s H_{\mathrm{NM}}} \psi$ are strongly continuous in $s \in \mathbb{R}$.

Then the Heisenberg operators $q_{j \mu}(t), p_{j \mu}(t), \phi(t, f)$ and $\pi(t, f)\left(f \in \mathcal{S}_{\text {real }}\left(\mathbb{R}^{d}\right)\right)$ are weakly differentiable on $\mathcal{E}$ and

$$
\begin{aligned}
\mathrm{w}-\left.\frac{d q_{j \mu}(t)}{d t}\right|_{\mathcal{E}} & =\frac{p_{j \mu}(t)}{m_{j}} \\
\mathrm{w}-\left.\frac{d p_{j \mu}(t)}{d t}\right|_{\mathcal{E}} & =-D_{j \mu} V(q(t))-\lambda e^{i t H_{\mathrm{NM}}} \phi\left(D_{\mu} \rho_{j}\left(q_{j}-\cdot\right)\right) e^{-i t H_{\mathrm{NM}}}, \\
\mathrm{w}-\left.\frac{d \phi(t, f)}{d t}\right|_{\mathcal{E}} & =\pi(t, f), \\
\mathrm{w}-\left.\frac{d \pi(t, f)}{d t}\right|_{\mathcal{E}} & =-\phi\left(t, \omega(-i \nabla)^{2} f\right)-\lambda \sum_{j=1}^{N}\left(\rho_{j} * f\right)\left(q_{j}(t)\right),
\end{aligned}
$$

where $q_{j}(t):=\left(q_{j 1}(t), \cdots, q_{j d}(t)\right), q(t):=\left(q_{1}(t), \cdots, q_{N}(t)\right)$.

Proof. By (A.3) and the CCR

$$
\left[p_{j \mu}, q_{l \nu}\right]=-i \delta_{j l} \delta_{\mu \nu} \quad \text { on } D\left(p_{j \mu} q_{l \nu}\right) \cap D\left(q_{l \nu} p_{j \mu}\right)
$$

we have

$$
\left[H_{\mathrm{p}}, q_{j \mu}\right]_{\mathrm{w}}^{\mathcal{E}}=-i \frac{p_{j \mu}}{m_{j}},
$$

which, together with (N.5), implies that

$$
\left[H_{\mathrm{NM}}, q_{j \mu}\right]_{\mathrm{w}}^{\mathcal{E}}=-i \frac{p_{j \mu}}{m_{j}} .
$$


By this fact, (A.4) and (A.5), we can apply Lemma 2.7 to obtain (2.36).

Let

$$
h_{0}:=-\sum_{j=1}^{N} \frac{\Delta_{q_{j}}}{2 m_{j}} .
$$

For all $\psi \in D\left(h_{0}\right)$, we have

$$
\begin{aligned}
\left\|p_{j \mu} \psi\right\|^{2} & \leq 2 m_{j}\left\langle\psi, h_{0} \psi\right\rangle \leq 2 m_{j}\|\psi\|\left\|h_{0} \psi\right\| \\
& \leq \varepsilon\left\|h_{0} \psi\right\|^{2}+\frac{m_{j}^{2}}{\varepsilon}\|\psi\|^{2},
\end{aligned}
$$

where $\varepsilon>0$ is arbitrary. By the self-adjointness of $H_{\mathrm{p}}$ and the closed graph theorem, there exist constants $a \geq 0, b \geq 0$ such that, for all $\psi \in D\left(H_{\mathrm{p}}\right)=D\left(h_{0}\right) \cap D(V)$,

$$
\left\|h_{0} \psi\right\|+\|V \psi\| \leq a\left\|H_{\mathrm{p}} \psi\right\|+b\|\psi\|
$$

Hence, by (2.42),

$$
\left\|p_{j \mu} \psi\right\| \leq a \sqrt{\varepsilon}\left\|H_{\mathrm{p}} \psi\right\|+\left(b \sqrt{\varepsilon}+\frac{m_{j}}{\sqrt{\varepsilon}}\right)\|\psi\| .
$$

Let $\psi, \phi \in C_{0}^{\infty}\left(\mathbb{R}^{d N}\right)$. Then we have

$$
\left\langle H_{\mathrm{p}} \psi, p_{j \mu} \phi\right\rangle-\left\langle p_{j \mu} \psi, H_{\mathrm{p}} \phi\right\rangle=\left\langle\psi, i\left(D_{j \mu} V\right) \phi\right\rangle .
$$

By (A.1), (A.2) and (2.44), we can extend this equality to all $\psi, \phi \in D\left(H_{\mathrm{p}}\right)$. Hence, in particular, we have

$$
\left[H_{\mathrm{p}}, p_{j \mu}\right]_{\mathrm{w}}^{\mathcal{E}}=i D_{j \mu} V
$$

in the sense of quadratic form on $\mathcal{E}$. Hence, by (N.5), we have

$$
\left[H_{\mathrm{NM}}, p_{j \mu}\right]_{\mathrm{w}}^{\mathcal{E}}=i D_{j \mu} V+i \lambda \phi\left(D_{\mu} \rho_{j}\left(q_{j}-\cdot\right)\right) \text {. }
$$

in the sense of quadratic form on $\mathcal{E}$. By this fact, (A.4) and (A.6), we can apply Lemma 2.7 to obtain (2.37). Similarly (more easily) we can prove (2.38) and (2.39).

now we show that Theorem 2.8 can be applied to the SNM.

Lemma 2.9 Let $S$ be a self-adjoint operator on a Hilbert space $\mathcal{X}$ and $A$ be a linear operator on $\mathcal{X}$ such that, for some $\alpha>0, A$ is $|S|^{\alpha}$-bounded, i.e., $D\left(|S|^{\alpha}\right) \subset D(A)$ and

$$
\|A \psi\| \leq a\left\||S|^{\alpha} \psi\right\|+b\|\psi\|, \quad \psi \in D\left(|S|^{\alpha}\right)
$$

where $a, b \geq 0$ are constants. Then, for all $\psi \in D\left(|S|^{\alpha}\right)$, the function: $t \rightarrow A e^{i t S} \psi$ is strongly continuous.

Proof. We have for all $h \in \mathbb{R}$

$$
\begin{aligned}
\left\|A e^{i(t+h) S} \psi-A e^{i t S} \psi\right\| & \leq a\left\||S|^{\alpha}\left(e^{i h S}-1\right) e^{i t S} \psi\right\|+b\left\|\left(e^{i h S}-1\right) \psi\right\| \\
& =a\left\|\left(e^{i h S}-1\right)|S|^{\alpha} \psi\right\|+b\left\|\left(e^{i h S}-1\right) \psi\right\|,
\end{aligned}
$$


which implies the strong continuity of the function: $\rightarrow A e^{i t S} \psi$.

For a self-adjoint operator $T$ on a Hilbert space which is bounded from below, we set

$$
E_{0}(T):=\inf \sigma(T)
$$

the ground state energy of $T$, where $\sigma(T)$ denotes the spectrum of $T$. Under hypotheses (H.1) and (H.2), we set

$$
\hat{H}_{\mathrm{SNM}}:=H_{\mathrm{SNM}}-E_{0}\left(H_{\mathrm{SNM}}\right)
$$

which is nonnegative.

Lemma 2.10 Assume (H.1) and (H.2). Suppose that $Q(V) \subset D(|q|)$ and there exist constants $c_{1}, c_{2} \geq 0$ such that for all $u \in Q(V)$

$$
\||q| u\| \leq c_{1}\left\||V|^{1 / 2} u\right\|+c_{2}\|u\| .
$$

Then, for all $j=1, \cdots, N, \mu=1, \cdots, d$ and $\psi \in Q\left(H_{\mathrm{SNM}}\right)$, the $\mathcal{H}$-valued function $q_{j \mu} e^{i s H_{\mathrm{SNM}}} \psi$ is strongly continuous in $s \in \mathbb{R}$.

Proof. By Proposition 2.1 and the closed graph theorem, there exist constants $a_{1}, b_{1} \geq 0$ such that, for all $\psi \in D\left(H_{\mathrm{SNM}}\right)=D\left(H_{0}\right)$,

$$
\left\|H_{\mathrm{p}} \psi\right\|+\left\|H_{\mathrm{b}} \psi\right\| \leq a_{1}\left\|H_{\mathrm{SNM}} \psi\right\|+b_{1}\|\psi\| .
$$

By this inequality and (2.43), we have

$$
\|V \psi\| \leq a a_{1}\left\|H_{\mathrm{SNM}} \psi\right\|+\left(a b_{1}+b\right)\|\psi\|,
$$

which, via an application [11, Theorem X.18], implies that $Q\left(H_{\mathrm{SNM}}\right) \subset Q(V)$ and

$$
\left\||V|^{1 / 2} \phi\right\| \leq a_{2}\left\|\hat{H}_{\mathrm{SNM}}^{1 / 2} \phi\right\|+b_{2}\|\phi\|, \quad \phi \in Q\left(H_{\mathrm{SNM}}\right),
$$

with $a_{2}, b_{2} \geq 0$ being constants. Hence, by (2.49), we obtain

$$
\left\|q_{j \mu} \psi\right\| \leq c_{1} a_{2}\left\|\hat{H}_{\mathrm{SNM}}^{1 / 2} \psi\right\|+\left(c_{1} b_{2}+c_{2}\right)\|\psi\|, \quad \psi \in Q\left(H_{\mathrm{SNM}}\right) .
$$

Hence we can apply Lemma 2.9 to obtain the desired result.

Lemma 2.11 Assume (H.1) and (H.2). Suppose that

$$
\mathcal{S}_{\text {real }}\left(\mathbb{R}^{d}\right) \subset H_{\omega}^{-1} \cap H_{\omega}^{1 / 2} .
$$

Let $\psi \in Q\left(H_{\mathrm{SNM}}\right)$. Then, for all $f \in \mathcal{S}_{\text {real }}\left(\mathbb{R}^{d}\right)$, the $\mathcal{H}$-valued functions $\phi_{\mathrm{F}}(f) e^{\text {is }_{\mathrm{SNM}}} \psi$ and $\pi_{\mathrm{F}}(f) e^{i s H_{\mathrm{SNM}}} \psi$ are strongly continuous in $s \in \mathbb{R}$.

Proof. Using the well-known estimates

$$
\begin{aligned}
\|a(f) \psi\| & \leq\left\|\omega^{-1 / 2} f\right\|_{L^{2}\left(\mathbb{R}^{d}\right)}\left\|H_{\mathrm{b}}^{1 / 2} \psi\right\|, \\
\left\|a(f)^{*} \psi\right\| \leq & \leq \omega^{-1 / 2} f\left\|_{L^{2}\left(\mathbb{R}^{d}\right)}\right\| H_{\mathrm{b}}^{1 / 2} \psi\|+\| f \|_{L^{2}\left(\mathbb{R}^{d}\right)}, \\
& \psi
\end{aligned}
$$


we have

$$
\begin{gathered}
\left\|\phi_{\mathrm{F}}(f) \psi\right\| \leq \sqrt{2}\left\|\omega^{-1} \hat{f}\right\|_{L^{2}\left(\mathbb{R}^{d}\right)}\left\|H_{\mathrm{b}}^{1 / 2} \psi\right\|+\frac{1}{\sqrt{2}}\left\|\omega^{-1 / 2} \hat{f}\right\|_{L^{2}\left(\mathbb{R}^{d}\right)}\|\psi\|, \\
f \in H_{\omega}^{-1} \cap H_{\omega}^{-1 / 2}, \\
\left\|\pi_{\mathrm{F}}(g) \psi\right\| \leq \sqrt{2}\|\hat{g}\|_{L^{2}\left(\mathbb{R}^{d}\right)}\left\|H_{\mathrm{b}}^{1 / 2} \psi\right\|+\frac{1}{\sqrt{2}}\left\|\omega^{1 / 2} \hat{g}\right\|_{L^{2}\left(\mathbb{R}^{d}\right)}\|\psi\|, \\
g \in L^{2}\left(\mathbb{R}^{d}\right) \cap H_{\omega}^{1 / 2} .
\end{gathered}
$$

By (2.50) and an application [11, Theorem X.18], we have

$$
\left\|H_{\mathrm{b}}^{1 / 2} \psi\right\| \leq a_{3}\left\|\hat{H}_{\mathrm{SNM}}^{1 / 2} \psi\right\|+b_{3}\|\psi\|, \quad \psi \in Q\left(H_{\mathrm{SNM}}\right)
$$

with $a_{3}, b_{3} \geq 0$ being constants. It follows that, for all $f \in \mathcal{S}_{\text {real }}\left(\mathbb{R}^{d}\right), \phi_{\mathrm{F}}(f)$ and $\pi_{\mathrm{F}}(f)$ are $\hat{H}_{\mathrm{SNM}}^{1 / 2}$-bounded. Hence an application of Lemma 2.9 yields the desired result. $\mathbf{\square}$ We shall need the following condition too.

(H.3) The function $\omega$ is such that

$$
\mathcal{S}_{\text {real }}\left(\mathbb{R}^{d}\right) \subset H_{\omega}^{-1} \cap H_{\omega}^{3 / 2}
$$

Theorem 2.12 Assume (H.1)-(H.3), (A.1), (A.2), (2.29) and (2.49). Suppose that

$$
D\left(H_{\mathrm{p}}\right) \subset \cap_{j, l=1}^{N} \cap_{\mu, \nu=1}^{d}\left[D\left(p_{j \mu} q_{l \nu}\right) \cap D\left(q_{l \nu} p_{j \mu}\right)\right] \cap\left(\cap_{j=1}^{N} \cap_{\mu=1}^{d} Q\left(D_{j \mu} V\right)\right)
$$

Then the conclusion of Theorem 2.8 holds with $H_{\mathrm{NM}}=H_{\mathrm{SNM}}, \phi(f)=\phi_{\mathrm{F}}(f), \pi(f)=\pi_{\mathrm{F}}(f)$ and $\mathcal{E}=D\left(H_{\mathrm{SNM}}\right)$.

Proof. We need only check that the assumption of Theorem 2.8 is satisfied with $H_{\mathrm{NM}}=$ $H_{\mathrm{SNM}}, \phi(f)=\phi_{\mathrm{F}}(f), \pi(f)=\pi_{\mathrm{F}}(f)$ and $\mathcal{E}=D\left(H_{\mathrm{SNM}}\right)$. (A.3) and (A.4) follow from (2.58). (A.5) and (A.6) follow from Lemmas 2.10 and 2.11 respectively.

\section{A Nelson model in a non-Fock representation}

In this section we define a Nelson model whose time-zero fields are given by a non-Fock representation of the CCR and show that it has a ground state even if no infrared cutoff is made.

Under hypotheses (H.2) and (H.3), we can define for each $f \in \mathcal{S}_{\text {real }}\left(\mathbb{R}^{d}\right)$

$$
\widetilde{\phi}(f):=\phi_{\mathrm{F}}(f)+\lambda \sum_{j=1}^{N} \int_{\mathbb{R}^{d}} \frac{\hat{\rho}_{j}(k) \hat{f}(k)}{\omega(k)^{2}} d k .
$$

We set

$$
\widetilde{\pi}(f):=\pi_{\mathrm{F}}(f) .
$$

Proposition 3.1 Assume (H.2) and (H.3). Then: 
(i) $\left\{\mathcal{F}_{\mathrm{b}}, \mathcal{F}_{0},\left\{\widetilde{\phi}(f), \widetilde{\pi}(f) \mid \mathcal{S}_{\text {real }}\left(\mathbb{R}^{d}\right)\right\}\right\}$ is a representation of the $C C R$ indexed by $\mathcal{S}_{\text {real }}\left(\mathbb{R}^{d}\right)$.

(ii) The representation $\left.\left\{\tilde{\phi}(f), \tilde{\pi}(f) \mid \mathcal{S}_{\text {real }}\left(\mathbb{R}^{d}\right)\right\}\right\}$ is unitarily equivalent to the Fock representation $\left.\left\{\phi_{\mathrm{F}}(f), \pi_{\mathrm{F}}(f) \mid \mathcal{S}_{\text {real }}\left(\mathbb{R}^{d}\right)\right\}\right\}$ if and only if $\sum_{j=1}^{N} \rho_{j} \in H_{\omega}^{-3 / 2}$.

Proof. Part (i) is obvious. Part (ii) follows from an argument similar to that in [5, Chapter $1, \S 1-\mathrm{e}]$.

We now consider a Nelson model whose time-zero fields are given by $\{\widetilde{\phi}(f), \widetilde{\pi}(f) \mid f \in$ $\mathcal{S}_{\text {real }}\left(\mathbb{R}^{d}\right)$. We introduce an $L^{2}\left(\mathbb{R}^{d}\right)$-valued function $G$ on $\mathbb{R}^{d N}\left(G: \mathbb{R}^{d N} \rightarrow L^{2}\left(\mathbb{R}^{d}\right)\right.$, $\left.G(q) \in L^{2}\left(\mathbb{R}^{d}\right), q \in \mathbb{R}^{d N}\right)$ by

$$
G(q)(k):=\sum_{j=1}^{N} \frac{\hat{\rho}_{j}(k)^{*}}{\sqrt{\omega(k)}}\left(e^{-i q_{j} k}-1\right)
$$

and a function

$$
W(q):=\sum_{j, l=1}^{N} \int_{\mathbb{R}^{d}} \frac{\hat{\rho}_{j}(k) \hat{\rho}_{l}(k)^{*}}{\omega(k)^{2}} e^{-i q_{l} k} d k, \quad q \in \mathbb{R}^{d N} .
$$

By reality of $\rho_{j}$ and (2.3), $W$ is real-valued. We define a Hamiltonian by

$$
H:=H_{0}+\lambda \Phi_{\mathrm{S}}(G(q))-\lambda^{2} W+c_{0} \lambda^{2},
$$

where

$$
c_{0}:=\frac{1}{2}\left\|\frac{\sum_{j=1}^{N} \hat{\rho}_{j}}{\omega}\right\|_{L^{2}\left(\mathbb{R}^{d}\right)}^{2} .
$$

Lemma 3.2 Assume (H.1)-(H.3). Then $H$ is self-adjoint with $D(H)=D\left(H_{0}\right)$ and bounded from below. Moreover, $H$ is essentially self-adjoint on each core of $H_{0}$.

Proof. In the same way as in the case of $H_{\mathrm{SNM}}$, we can show that $H^{\prime}:=H_{0}+\lambda \Phi_{\mathrm{S}}(G(q))$ is self-adjoint with $D\left(H^{\prime}\right)=D\left(H_{0}\right)$, bounded from below, and essentially self-adjoint on each core of $H_{0}$. Since $-\lambda^{2} W+c_{0} \lambda^{2}$ is a bounded self-adjoint operator, the Kato-Rellich theorem yields the desired result.

Let

$$
L_{\lambda}^{\mathrm{NF}}:=H_{\mathrm{b}}+\lambda \Phi_{\mathrm{S}}(G(q))-\lambda^{2} W+c_{0} \lambda^{2}
$$

so that

$$
H=H_{\mathrm{p}}+L_{\lambda}^{\mathrm{NF}}
$$

Proposition 3.3 Assume (H.1)-(H.3). Then

$$
\mathbb{M}_{\mathrm{NF}}:=\left\{\mathcal{H}, \mathcal{D}_{0}, L_{\lambda}^{\mathrm{NF}},\left\{\widetilde{\phi}(f), \tilde{\pi}(g) \mid f \in H_{\omega}^{-1}, g \in H_{\omega}^{1 / 2}\right\}\right\}
$$

is a Nelson model.

Proof. Similar to the proof of Proposition 2.4. 
Proposition 3.4 Assume (H.1)-(H.3). Then $\left\{H,\left\{\widetilde{\phi}(f), \widetilde{\pi}(f) \mid f \in \mathcal{S}_{\text {real }}\left(\mathbb{R}^{d}\right)\right\}\right\}$ is unitarily equivalent to $\left\{H_{\mathrm{SNM}},\left\{\phi_{\mathrm{F}}(f), \pi_{\mathrm{F}}(f) \mid f \in \mathcal{S}_{\text {real }}\left(\mathbb{R}^{d}\right)\right\}\right\}$ if and only if

$$
\sum_{j=1}^{N} \rho_{j} \in H_{\omega}^{-3 / 2}
$$

Proof. Suppose that there exists a unitary operator $Y$ on $\mathcal{H}$ such that

$$
\begin{aligned}
& Y H Y^{-1}=H_{\mathrm{SNM}}, \\
& Y \widetilde{\phi}(f) Y^{-1}=\phi_{\mathrm{F}}(f), Y \widetilde{\pi}(f) Y^{-1}=\pi_{\mathrm{F}}(f), \quad f \in \mathcal{S}_{\text {real }}\left(\mathbb{R}^{d}\right) .
\end{aligned}
$$

Then, by Proposition 3.1, (3.10) holds. Conversely, suppose that (3.10) holds. Then we can define a unitary operator $U$ by

$$
U:=\exp \left(-i \lambda \Phi_{\mathrm{S}}\left(i \frac{\sum_{j=1}^{N} \hat{\rho}_{j}^{*}}{\omega^{3 / 2}}\right)\right) .
$$

It is easy to check that (3.11) and (3.12) hold with $Y=U$.

-Proposition 3.4 shows that, under (H.1)-(H.3) and the condition that $\sum_{j=1}^{N} \rho_{j} \in H_{\omega}^{-3 / 2}$, the Nelson model $\mathbb{M}_{\mathrm{NF}}$ is equivalent to the SNM. In this case, under suitable additional conditions, $H_{\mathrm{SNM}}$ has a ground state [7] and so does $H$. Thus we are interested in the case

$$
\sum_{j=1}^{N} \rho_{j} \notin H_{\omega}^{-3 / 2} .
$$

This condition is called an infrared singularity condition. In this paper, we say that the Nelson model $\mathbb{M}_{\text {Nelson }}$ has no infrared cutoff if (3.14) holds. Under condition (3.14), the Nelson model $\mathbb{M}_{\mathrm{NF}}$ is not unitarily equivalent to the SNM. Note that (3.14) and the natural condition $\sum_{j=1}^{N} \rho_{j} / \sqrt{\omega} \in L^{2}\left(\mathbb{R}^{d}\right)$ imply

$$
\operatorname{ess}_{\inf } \operatorname{s\in \mathbb {R}}^{d} \omega(k)=0
$$

("ess.inf" means essential infimum), i.e., the quantum scalar field under consideration is "massless".

\section{Existence of a ground state of the Nelson model $\mathrm{M}_{\mathrm{NF}}$ without infrared cutoff}

Let $T$ be a self-adjoint operator on a Hilbert space and bounded from below. We say that $T$ has a ground state if there exists a non-zero vector $\psi \in D(T)$ such that $T \psi=E_{0}(T) \psi$. In this case $\psi$ is called a ground state of $T$. We show below that, under suitable conditions, the Hamiltonian $H$ of the Nelson model $\mathbb{M}_{N F}$ has a ground state even in the case where it has no infrared cutoff (i.e., (3.14) holds). We formulate some additional conditions: 
(H.4) The function $\omega$ is continuous on $\mathbb{R}^{d}$ satisfying (3.15) and the following conditions:

$$
\begin{aligned}
& D_{\mu} \omega \in L^{\infty}\left(\mathbb{R}^{d}\right), \mu=1, \cdots, d, \\
& \lim _{|k| \rightarrow \infty} \omega(k)=\infty
\end{aligned}
$$

(H.5) For $j=1, \cdots, N$,

$$
\int_{\mathbb{R}^{d}} \frac{|k|^{2}\left|\hat{\rho}_{j}(k)\right|^{2}}{\omega(k)^{3}} d k<\infty
$$

(H.6) For all $R>0, \int_{|q| \leq R}|V(q)| d q<\infty$ and there exist constants $c_{1}, c_{2} \geq 0$ such that

$$
|q|^{2} \leq c_{1} V(q)+c_{2}, \quad \text { a.e. } q \in \mathbb{R}^{d N} .
$$

An infrared-cutoff Hamiltonian of the SNM is defined by

$$
H_{\mathrm{SNM}, \sigma}:=H_{0}+\lambda \sum_{j=1}^{N} \Phi_{\mathrm{S}}\left(e^{-i k q_{j}} \frac{\chi_{\omega \geq \sigma} \hat{\rho}_{j}^{*}}{\sqrt{\omega}}\right),
$$

where $\sigma>0$ is an infrared cutoff parameter and $\chi_{S}$ is a characteristic function of the set $S$.

Under (H.2), we can define for all $\sigma>0$ a unitary operator

$$
U_{\sigma}:=\exp \left(-i \lambda \Phi_{\mathrm{S}}\left(i \frac{\sum_{j=1}^{N} \chi_{\omega \geq \sigma} \hat{\rho}_{j}}{\omega^{3 / 2}}\right)\right) .
$$

Theorem 4.1 Assume (H.1), (H.2) and (H.4)-(H.6). Then $H$ has a ground state $\psi_{0}$ which has the following property: there exists a sequence $\left\{\phi_{\sigma_{n}}\right\}_{n=1}^{\infty}$ of unit vectors in $D\left(H_{0}\right)$ such that $\sigma_{n}>0, n \in \mathbb{N}, \lim _{n \rightarrow \infty} \sigma_{n}=0$, each $\phi_{\sigma_{n}}$ is a ground state of $H_{\mathrm{SNM}, \sigma_{n}}$ and

$$
\mathrm{w}-\lim _{n \rightarrow \infty} U_{\sigma_{n}}^{-1} \phi_{\sigma_{n}}=\psi_{0}
$$

where "w-lim" means weak limit.

Remark 4.1 Consider the physical case $\omega=\omega_{0}((2.4)$ with $m=0)$. Hence (H.4) holds. Assume (H.2) with $\omega=\omega_{0}$ and that

$$
\sum_{j=1}^{N} \frac{\hat{\rho}_{j}(\cdot)}{|k|^{3 / 2}} \notin L^{2}\left(\mathbb{R}^{d}\right) .
$$

Then (3.14) and (H.5) hold with $\omega=\omega_{0}$. Hence Theorem 4.1 holds in the physical case without infrared cutoff.

Remark 4.2 Assume (3.14). Then we have

$$
\mathrm{W}-\lim _{\sigma \rightarrow 0} U_{\sigma}=0 \text {. }
$$

This is an expression of infrared divergence. Hence the relation w- $\lim _{n \rightarrow \infty} U_{\sigma_{n}}^{-1} \phi_{\sigma_{n}}=\psi_{0}$ in Theorem 4.1 suggests that $\mathrm{w}-\lim _{n \rightarrow \infty} \phi_{\sigma_{n}}=0$. 
The idea of proof of Theorem 4.1 is to apply [7, Theorem 1].

Let

$$
K:=H_{\mathrm{p}}-\lambda^{2} W+c_{0} \lambda^{2} .
$$

Lemma 4.2 Assume (H.1) and (H.2). Then $K$ is self-adjoint and bounded from below.

Proof. This follows from the boundedness of $\lambda^{2} W$ and a simple application of the KatoRellich theorem.

$$
\hat{K}:=K-E_{0}(K)
$$

Lemma 4.3 Assume (H.1), (H.2) and (H.6). Then $(\hat{K}+i)^{-1}$ is compact.

Proof. By (H.6) and the boundedness of $W$, we have

$$
\lim _{|q| \rightarrow \infty}\left\{V(q)-\lambda^{2} W(q)+c_{0} \lambda^{2}\right\}=\infty .
$$

Hence, applying [12, p.249, Theorem XIII.67], we obtain the desired result.

We denote by $\mathrm{B}\left(L^{2}\left(\mathbb{R}^{d N}\right)\right)$ the set of bounded linear operators on $L^{2}\left(\mathbb{R}^{d N}\right)$. For each $k \in \mathbb{R}^{d}$, we define a linear operator on $L^{2}\left(\mathbb{R}^{d N}\right)$ as follows:

$$
v(k):=\left\{\begin{array}{ll}
G(\cdot)(k) & \omega(k)>0 \\
0 & \omega(k)=0
\end{array} .\right.
$$

Remark 4.3 By the original assumption for $\omega$, the Lebesgue measure of the set $\{k \in$ $\left.\mathbb{R}^{d} \mid \omega(k)=0\right\}$ is equal to zero. Hence, $v(k)=G(\cdot)(k)$ a.e. $k \in \mathbb{R}^{d}$.

Lemma 4.4 Assume (H.2). Then, for a.e. $k \in \mathbb{R}^{d}, v(k) \in \mathrm{B}\left(L^{2}\left(\mathbb{R}^{d N}\right)\right)$ with

$$
\|v(k)\| \leq 2 \sum_{j=1}^{N} \frac{\left|\hat{\rho}_{j}(k)\right|}{\sqrt{\omega(k)}} .
$$

Proof. This easily follows from (3.3).

The following lemma immediately follows from Lemma 4.4.

Lemma 4.5 Assume (H.1) and (H.2). Then, for a.e. $k \in \mathbb{R}^{d}$,

$$
v(k)(\hat{K}+1)^{-1 / 2} \in \mathrm{B}\left(L^{2}\left(\mathbb{R}^{d N}\right), \quad(\hat{K}+1)^{-1 / 2} v(k) \in \mathrm{B}\left(L^{2}\left(\mathbb{R}^{d N}\right)\right) .\right.
$$

Lemma 4.6 Assume (H.1) and (H.2). Then, for all $u_{1}, u_{2} \in L^{2}\left(\mathbb{R}^{d N}\right)$, the functions

$$
\left(u_{2}, v(\cdot)(\hat{K}+1)^{-1 / 2} u_{1}\right),\left(u_{2},(\hat{K}+1)^{-1 / 2} v(\cdot) u_{1}\right),
$$

on $\mathbb{R}^{d}$ are measurable. Moreover,

$$
C(R):=\int_{\mathbb{R}^{d}} \frac{1}{\omega(k)}\left(\left\|v(k)(\hat{K}+R)^{-1 / 2}\right\|^{2}+\left\|(\hat{K}+R)^{-1 / 2} v(k)\right\|^{2}\right) d k<\infty
$$

and

$$
\lim _{R \rightarrow \infty} C(R)=0
$$


Proof. We have

$$
\left(u_{2}, v(k)(\hat{K}+1)^{-1 / 2} u_{1}\right)=\sum_{j=1}^{N} \frac{\hat{\rho}_{j}(k)^{*}}{\sqrt{\omega(k)}} g_{j}(k)
$$

with

$$
g_{j}(k)=\int_{\mathbb{R}^{d}} u_{2}(q)^{*}\left(e^{-i q_{j} k}-1\right)\left((\hat{K}+1)^{-1 / 2} u_{1}\right)(q) d q .
$$

It is easy to see that $g_{j}$ is continuous on $\mathbb{R}^{d}$. Hence $\left(u_{2}, v(\cdot)(\hat{K}+1)^{-1 / 2} u_{1}\right)$ is measurable. Similarly we can show that $\left(u_{2},(\hat{K}+1)^{-1 / 2} v(\cdot) u_{1}\right)$ is measurable.

We have

$$
C(R) \leq \frac{2}{R} \int_{\mathbb{R}^{d}} \frac{1}{\omega(k)}\|v(k)\|^{2} d k \leq \frac{8 N}{R} \sum_{j=1}^{N} \int_{\mathbb{R}^{d}} \frac{\left|\hat{\rho}_{j}(k)\right|^{2}}{\omega(k)^{2}} d k .
$$

Hence $C(R)<\infty$ and (4.8) holds.

Lemma 4.7 Assume (H.1) and (H.6). Let $\psi \in D\left(\hat{K}^{1 / 2}\right)$. Then $\psi \in D\left(\left|q_{j}\right|\right)$ and, for all $j=1, \cdots, N$,

$$
\left\|\left|q_{j}\right| \psi\right\| \leq c_{1}\left\|\hat{K}^{1 / 2} \psi\right\|+\left(c_{1} \lambda^{2}\|W\|+c_{1} c_{0} \lambda^{2}+c_{1} E_{0}(K)+c_{2}\right)\|\psi\|^{2} .
$$

In particular,

$$
Q_{j}:=\left|q_{j}\right|(\hat{K}+1)^{-1 / 2}
$$

is bounded.

Proof. Let $\psi \in D(K)$. Then, using (H.6), one can easily prove (4.9). Since $D(K)$ is a core of $\hat{K}^{1 / 2}$, it follows from a limiting argument that $D\left(\left|q_{j}\right|\right) \supset D\left(\hat{K}^{1 / 2}\right)$ and (4.9) holds for all $\psi \in D\left(\hat{K}^{1 / 2}\right)$.

Lemma 4.8 Assume (H.1), (H.2), (H.5) and (H.6). Then

$$
\int_{\mathbb{R}^{d}} \frac{1}{\omega(k)^{2}}\left\|v(k)(\hat{K}+1)^{-1 / 2}\right\|^{2} d k<\infty
$$

Proof. By the elementary inequality

$$
\left|e^{-i q_{j} k}-1\right| \leq\left|q_{j}\right||k|
$$

we have for all $u \in L^{2}\left(\mathbb{R}^{d N}\right)$

$$
\|v(k) u\| \leq \sum_{j=1}^{N} \frac{\left|\hat{\rho}_{j}(k)\right||k|}{\sqrt{\omega(k)}}\left\|\left|q_{j}\right| u\right\| .
$$

Hence

$$
\left\|v(k)(\hat{K}+1)^{-1 / 2}\right\| \leq \sum_{j=1}^{N} \frac{\left|\hat{\rho}_{j}(k)\right||k|}{\sqrt{\omega(k)}}\left\|Q_{j}\right\|,
$$

which, by (H.5), implies (4.11). 
Proof of Theorem 4.1 We have

$$
H=K \otimes I+I \otimes H_{\mathrm{b}}+H_{I}(v)
$$

with

$$
H_{I}(v):=\frac{\lambda}{\sqrt{2}} \int_{\mathbb{R}^{d}}\left(v(k) a(k)^{*}+v(k)^{*} a(k)\right) d k .
$$

Hence $H$ is exactly of the same form as the Hamiltonians considered in [7]. Lemmas 4.2-4.8 show that $H$ satisfies all the assumptions of [7, Theorem 1]. Thus we can apply [7, Theorem 1] to conclude that $H$ has a ground state $\psi_{0}$. ¿From the proof of $[7$, Theorem 1], we see that $\psi_{0}$ is a weak limit of a sequence $\left\{\psi_{\sigma_{n}}\right\}_{n=1}^{\infty}$ of unit vectors in $D\left(H_{0}\right)$ such that $\sigma_{n}>0, \lim _{n \rightarrow \infty} \sigma_{n}=0$ and each $\psi_{\sigma_{n}}$ is a ground state of the infrared-cutoff version $H_{\sigma_{n}}$ of $H$ :

$$
H_{\sigma}:=K \otimes I+I \otimes H_{\mathrm{b}}+H_{I}\left(v_{\sigma}\right), \quad \sigma>0,
$$

where $v_{\sigma}(k):=v(k) \chi_{\omega \geq \sigma}(k)$. By Proposition 3.4, $\phi_{\sigma_{n}}:=U_{\sigma_{n}} \psi_{\sigma_{n}}$ is a ground state of $H_{\mathrm{SNM}, \sigma_{n}}$ with $\left\|\phi_{\sigma_{n}}\right\|=1$. Hence (4.5) holds.

\section{References}

[1] A. Arai, Scaling limit for quantum systems of nonrelativistic particles interacting with a Bose field, Hokkaido University Preprint Series in Mathematics \#59, 1989.

[2] A. Arai, M. Hirokawa and F. Hiroshima, On the absence of eigenvectors of Hamiltonians in a class of massless quantum field models without infrared cutoff, J. Funct. Anal. 168(1999), 470-497.

[3] A. Arai and M. Hirokawa, Ground states of a general class of quantum field Hamiltonians, Rev. Math. Phys. 12 (2000), 1085-1135.

[4] V. Bach, J. Fröhlich and I. M. Sigal, Spectral analysis for systems of atoms and molecules coupled to the quantized radiation field, Commun. Math. Phys. 207(1998), 249-290.

[5] G. G. Emch, Algebraic Methods in Statistical Mechanics and Quantum Field Theory, John Wiley \& Sons, 1972.

[6] J. Fröhlich, On the infrared problem in a model of scalar electrons and massless scalar bosons, Ann. Inst. Henri Poincaré 19(1973), 1-103.

[7] C. Gérard, On the existence of ground states for massless Pauli-Fierz Hamiltonians, Ann. Henri Poincaré 1(2000), 443-459.

[8] M. Griesemer, E. H. Lieb and M. Loss, Ground states in non-relativistic quantum electrodynamics, preprint, 2000.

[9] J. Lörinczi, R. A. Minlos, and H. Spohn, The infrared behaviour in Nelson's model of a quantum particle coupled to a massless scalar field, preprint, 2000. 
[10] E. Nelson, Interaction of nonrelativistic particles with a quantized scalar field, J. Math. Phys. 5(1964), 1190-1197.

[11] M. Reed and B. Simon, Methods of Modern Mathematical Physics Vol. II: Fourier Analysis, Self-Adjointness, Academic Press, New York, 1975.

[12] M. Reed and B. Simon, Methods of Modern Mathematical Physics Vol. IV: Analysis of Operators, Academic Press, New York, 1978.

[13] H. Spohn, Ground state(s) of the spin-boson Hamiltonian, Commun. Math. Phys. 123 (1989), 277-304.

[14] H. Spohn, Ground state of a quantum particle coupled to a scalar Bose field, Lett. Math. Phys. 44 (1998), 9-16. 